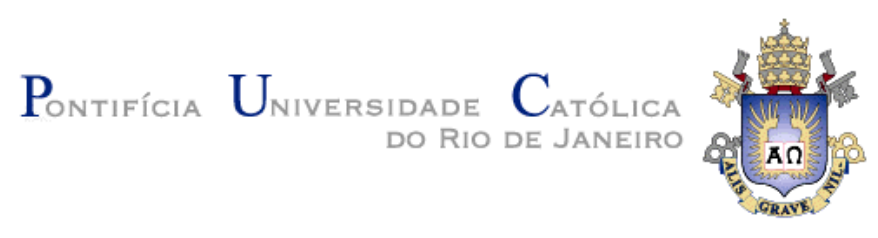

Ricardo Gomes Clemente

\title{
Uma arquitetura para processamento de eventos de log em tempo real
}

Dissertação de Mestrado

Dissertação apresentada como requisito parcial para obtenção do título de Mestre pelo Programa de PósGraduação em Informática da PUC-Rio.

Orientador: Prof. Marco Antonio Casanova 


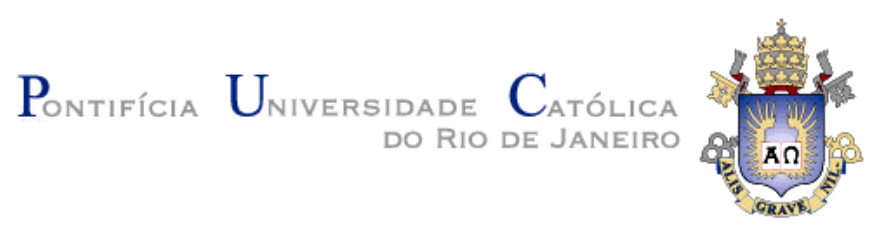

Ricardo Gomes Clemente

\section{Uma arquitetura para processamento de eventos de log em tempo real}

Dissertação apresentada como requisito parcial para obtenção do título de Mestre pelo Programa de PósGraduação em Informática da PUC-Rio. Aprovada pela Comissão Examinadora abaixo assinada.

Prof. Marco Antonio Casanova

Orientador

Departamento de Informática - PUC-Rio

Prof. Karin Koogan Breitman

Departamento de Informática - PUC-Rio

Prof. Marcelo Tílio M. Carvalho

TecGraf - PUC-Rio

Prof. José Eugenio Leal

Coordenador Setorial do Centro

Técnico Científico - PUC-Rio

Rio de Janeiro, 21 de agosto de 2008 
Todos os direitos reservados. É proibida a reprodução total ou parcial do trabalho sem autorização da universidade, do autor e do orientador.

\section{Ricardo Gomes Clemente}

Graduou-se em Engenharia Elétrica: Ênfase em Eletrônica pela UFRJ (Universidade Federal do Rio de Janeiro) em Julho de 2006. Trabalha com desenvolvimento e projeto de infra-estrutura de aplicações para Internet na Globo.com desde 2005.

Ficha Catalográfica

Clemente, Ricardo Gomes
Uma arquitetura para processamento de eventos
de log em tempo real / Ricardo Gomes Clemente ;
orientador: Marco Antonio Casanova. - 2008.
77 f. : il.(color.) ; 30 cm
Dissertação (Mestrado em Informática)-
Pontifícia Universidade Católica do Rio de Janeiro, Rio de
Janeiro, 2007.
Inclui bibliografia
1. Informática - Teses. 2. Log. 3. Sistemas de
gerenciamento de log. 4. Correlação de eventos. 5.
Arquitetura orientada a eventos. I. Casanova, Marco
Antonio. II. Pontifícia Universidade Católica do Rio de
Janeiro. Departamento de Informática. III. Título.




\section{Agradecimentos}

Agradeço primeiramente a Deus.

Agradeço ao meu orientador, Prof. Casanova, pela sua imensa capacidade de ensinar e orientar através de longas e agradáveis conversas.

Agradeço à PUC-Rio pela bolsa concedida para a realização desta pesquisa.

Aos demais professores e funcionários da pós-graduação com quem tive a oportunidade de conviver nestes dois anos, em especial ao Prof. Luiz Fernando, com quem iniciei minha pesquisa.

Agradeço à Globo.com por incentivar e apoiar meus estudos concedendo tempo de trabalho para minha dedicação ao mestrado.

Aos meus pais, minha família e minha namorada por me darem apoio e motivação sempre. 


\section{Resumo}

Clemente, Gomes Ricardo; Casanova, Marco Antonio. Uma arquitetura para processamento de eventos de log em tempo real. Rio de Janeiro, 2008. 77p. Dissertação de Mestrado - Departamento de Informática, Pontifícia Universidade Católica do Rio de Janeiro.

Logs são, atualmente, riquíssima fonte de informação para administradores de sistemas e analistas de negócio. Em ambientes com grande volume de acesso e infra-estrutura de centenas de servidores, processar toda a informação gerada e correlacioná-la com o objetivo de identificar situações de interesse técnico e de negócio em tempo real, é considerado um grande desafio. Nesse sentido, são explicados tanto os conceitos relacionados aos arquivos de $\log$ e aos sistemas que se propõem a gerenciá-los, quanto os métodos e ferramentas de correlação de eventos em tempo real, para que, então, seja proposta uma arquitetura de sistema capaz de lidar com o desafio citado. Por fim, um protótipo é desenvolvido e uma prova de conceito baseada em um caso real de uso é realizada.

\section{Palavras-chave}

$\log$, sistemas de gerenciamento de log, correlação de eventos, arquitetura orientada a eventos. 


\section{Abstract}

Clemente, Gomes Ricardo; Casanova, Marco Antonio. An architecture for real time log events processing. Rio de Janeiro, 2008. 77p. MSc. Dissertation - Departamento de Informática, Pontifícia Universidade Católica do Rio de Janeiro.

Logs are, nowadays, a rich source of information for system administrators and business analysts. In environments with a high access volume and hundreds of servers, to process every generated information and correlate it, in order to identify interesting technical and business situations in real time, is considered a challenge. Considering that, concepts related to log files and systems that aim to manage it, besides methods and tools for real time event correlation are presented, in order to propose a system architecture capable of overcoming the stated challenge. At last, a prototype is developed and a concept prove based on a real case is done.

\section{Keywords}

$\log$, log management systems, event correlation, event-driven architecture 


\section{Sumário}

1 Introdução 12

1.1 Motivação 12

$\begin{array}{ll}1.2 \text { Proposta da dissertação } & 13\end{array}$

$\begin{array}{ll}1.3 \text { Organização da dissertação } & 13\end{array}$

2 Gerenciamento de Log 15

2.1 Definições básicas 15

2.2 Logs como um fluxo de eventos 16

$\begin{array}{ll}2.3 \text { Caracterização e padrões de logs } & 17\end{array}$

2.4 Sistema de gerenciamento de logs 21

3 Eventos: definição e processamento 24

3.1 Definição de eventos 24

3.2 Processamento de eventos 25

3.3 Cenários de uso para o Processamento Complexo de Eventos 28

3.4 Processadores complexos de eventos 29

3.4.1 Processadores baseados em regras 29

3.4.2 Processadores baseado em consultas 30

4 Proposta de arquitetura $\quad 35$

4.1 Requisitos de um Sistema de Gerenciamento de Logs 35

4.1.1 Requisitos Funcionais 35

4.1.2 Requisitos Não-funcionais $\quad 37$

4.2 Conceito de Arquitetura Orientada a Eventos 38

4.3 Arquitetura Orientada a Eventos para Gerenciamento de Eventos de $\log$ 
5.1 Visão geral $\quad 47$

5.2 Canal Principal de Comunicação de Eventos 48

5.3 Nó de primeira interação $\quad 50$

5.4 Nó de correlação de eventos por consulta 55

5.5 Um caso de uso do protótipo 57

5.6 Testes funcionais do protótipo 62

6 Conclusão $\quad 71$

$\begin{array}{ll}7 \text { Referências } & 73\end{array}$ 


\section{Lista de figuras}

Figura 1 - Logs como fluxos de eventos $\quad 17$

Figura 2 - Exemplo de registro de evento de log no padrão Common Logfile Format 19

Figura 3 - Diagrama ilustrando os conceitos relacionados a eventos 28

Figura 4- Comparação entre modelos 31

Figura 5 - Estágios de interações fluxo de eventos 39

Figura 6 - Canal Principal de Comunicação de Eventos, grupos e Nós. 41

Figura 7 - Composição de um Nó 42

Figura 8 - Arquitetura proposta e seus componentes 43

Figura 9- Exemplo de configuração de segmento no Spread 49

Figura 10 - Configuração LogPP 51

Figura 11- Exemplo de regra no SEC 53

Figura 12 - Componentes do Nó de primeira interação 55

Figura 13 - Descrição de um fluxo de eventos de log 56

Figura 14 - Exemplo de consulta contínua $\quad 57$

Figura 15 - Componentes do Nó de correlação de eventos por consulta 57

Figura 16 - Arquitetura de uma aplicação Web 59

Figura 17 - Filtros configurados no LogPP para prova de conceito 60

Figura 18 - Regras do SEC utilizadas no protótipo $\quad 61$

Figura 19 - Consultas utilizadas no protótipo 62 


\section{Lista de tabelas}

Tabela 1 - Níveis de severidade para eventos de log 18

Tabela 2 - Descrição de campos do Common Logfile Format 19

Tabela 3 - Diferentes formatos de mensagens de log com log $4 j \quad 20$

Tabela 4 - Diferentes formatos de eventos de log 45

Tabela 5 - Caso de teste Apache e LogPP 63

Tabela 6 - Caso de teste Apache e LogPP com vírgula 63

Tabela 7 - Caso de teste JbossWeb e LogPP 64

Tabela 8 - Caso de teste do SEC com padrão $404 \quad 65$

Tabela 9 - Caso de teste do SEC com erro específico 66

Tabela 10 - Caso de teste de fila de mensagens $\quad 67$

Tabela 11 - Caso de teste do Publicador 68

Tabela 12 - Caso de teste do Consumidor $\quad 69$

Tabela 13 - Caso de teste do Insersor $\quad 70$

Tabela 14 - Caso de teste do sistema completo 70 


\title{
Lista de siglas
}

\author{
API Application Program Interface \\ BAM Business Activity Monitoring \\ CEP Complex Event Processing \\ CLF Common Log Format \\ CSV Comma Separated Values \\ DSMS Data Stream Management System \\ EDA Event Driven Architecture \\ EPN Event Process Network \\ ESP Event Stream Processing \\ FIFO First-in First-out \\ HTTP Hypertext Transfer Protocol \\ IPC Inter-Process Communication \\ LMS Log Management System \\ RFC Request for Comments \\ SEC Simple Event Correlator \\ SQL Structured Query Language \\ XML Extensible Markup Language \\ XMPP Extensible Messaging and Presence Protocol
}

